Western University

Scholarship@Western

Brain and Mind Institute Researchers'

Publications

Brain and Mind Institute

$1-1-2014$

\title{
Short-duration stimulation of the supplementary eye fields perturbs anti-saccade performance while potentiating contralateral head orienting.
}

\author{
Brendan B Chapman \\ Graduate program in Neuroscience, The University of Western Ontario, London, ON, Canada \\ Brian D Corneil \\ Department of Physiology \& Pharmacology, The University of Western Ontario, London, ON, Canada \& \\ Department of Psychology, The University of Western Ontario, London, ON, Canada \& Robarts Research \\ Institute, London, ON N6A 5K8, Canada
}

Follow this and additional works at: https://ir.lib.uwo.ca/brainpub

Part of the Neurosciences Commons, and the Psychology Commons

Citation of this paper:

Chapman, Brendan B and Corneil, Brian D, "Short-duration stimulation of the supplementary eye fields perturbs anti-saccade performance while potentiating contralateral head orienting." (2014). Brain and Mind Institute Researchers' Publications. 128.

https://ir.lib.uwo.ca/brainpub/128 


\title{
Short-duration stimulation of the supplementary eye fields perturbs anti-saccade performance while potentiating contralateral head orienting
}

\author{
Brendan B. Chapman ${ }^{1}$ and Brian D. Corneil ${ }^{2,3,4}$ \\ ${ }^{1}$ Graduate program in Neuroscience, The University of Western Ontario, London, ON, Canada \\ ${ }^{2}$ Department of Physiology \& Pharmacology, The University of Western Ontario, London, ON, Canada \\ ${ }^{3}$ Department of Psychology, The University of Western Ontario, London, ON, Canada \\ ${ }^{4}$ Robarts Research Institute, London, ON N6A 5K8, Canada
}

Keywords: context, eye movements, frontal cortex, monkey, orienting

\begin{abstract}
Many forms of brain stimulation utilize the notion of state dependency, whereby greater influences are observed when a given area is more engaged at the time of stimulation. Here, by delivering intracortical microstimulation (ICMS) to the supplementary eye fields (SEF) of monkeys performing interleaved pro- and anti-saccades, we show a surprising diversity of state-dependent effects of ICMS-SEF. Short-duration ICMS-SEF passed around cue presentation selectively disrupted anti-saccades by increasing reaction times and error rates bilaterally, and also recruited neck muscles, favoring contralateral head turning to a greater degree on anti-saccade trials. These results are consistent with the functional relevance of the SEF for anti-saccades. The multiplicity of stimulation-evoked effects, with ICMS-SEF simultaneously disrupting anti-saccade performance and facilitating contralateral head orienting, probably reflects both the diversity of cortical and subcortical targets of SEF projections, and the response of this oculomotor network to stimulation. We speculate that the bilateral disruption of anti-saccades arises via feedback loops that may include the thalamus, whereas neck muscle recruitment arises via feedforward polysynaptic pathways to the motor periphery. Consideration of both sets of results reveals a more complete picture of the highly complex and multiphasic response to ICMSSEF that can play out differently in different effector systems.
\end{abstract}

\section{Introduction}

Stimulation remains a central tool for cognitive neuroscience. The effects of many forms of brain stimulation are dependent on the behavioral state at the time of stimulation (Pascual-Leone et al., 2000; Cohen \& Newsome, 2004), enabling inference of an area's activity or critical time of contribution to a task based on the effects of stimulation on behavior. Such state-dependent effects can be quite variable, with stimulation facilitating behavior in some instances and disrupting or delaying behaviors in others. For example, electrical intracortical microstimulation of the supplementary eye fields (ICMS-SEF) of monkeys evokes saccades (Schlag \& Schlag-Rey, 1987), doing so at progressively lower current thresholds as the animal prepares to move (Tehovnik et al., 1999). However, low-current ICMS-SEF delays self-timed but not conventional memory-guided saccades (Kunimatsu \& Tanaka, 2012), and delays visually guided saccades when the animal is performing a stop-signal task that occasionally requires the saccade cancellation (Stuphorn $\&$ Schall, 2006). These results attest to the causal contribution of the SEF to more cognitively demanding tasks, presumably via the

Correspondence: Dr B. D. Corneil, ${ }^{4}$ Robarts Research Institute, as above.

E-mail: bcorneil@uwo.ca

Received 16 September 2013, accepted 30 September 2013 disruptive effects of ICMS-SEF on the network engaged by task demands, with greater delays reflecting a greater degree of involvement of the SEF at the time of stimulation.

Recent work shows that ICMS-SEF also evokes rapid and robust recruitment of a contralateral head-turning synergy on neck muscles that begins $\sim 30 \mathrm{~ms}$ after stimulation onset, preceding saccades by 40-70 ms (Chapman et al., 2012). Stimulation of many oculomotor areas evokes an earlier response on the neck vs. saccades, due to differences in the processing of premotor cephalomotor vs. oculomotor commands (Corneil et al., 2002; Elsley et al., 2007; Farshadmanesh et al., 2008). The response latency following ICMS-SEF suggests that recruitment arises via feedforward connections from the SEF to the oculomotor brainstem (perhaps via the frontal eye fields, FEFs), and then onto the motor periphery (Chapman et al., 2012). If so, larger evoked neck muscle responses should occur when the SEF are more active at the time of stimulation.

The question we ask is whether ICMS-SEF can simultaneously disrupt some aspects of oculomotor behavior (e.g. saccades) while facilitating others (e.g. neck muscle recruitment). Such a result would reveal novel perspectives about state dependency and its application in cognitive neuroscience, emphasizing the importance of considering how the effects of stimulation are assessed. Here, we investigate the effects of ICMS-SEF while monkeys performed 
interleaved pro- or anti-saccades, requiring them to look towards or away from a peripheral cue, respectively, depending on the color of the fixation point (Fig. 1A). SEF activity is greater on anti-saccade trials (Schlag-Rey et al., 1997; Amador et al., 2004), and hence we predict greater effects, whether disruptive or facilitatory, will accompany anti-saccades. Importantly, we employ very short-duration (30 ms) ICMS-SEF, which can robustly recruit neck muscles without directly evoking saccades, allowing the animal to continue to perform the task. Short-duration ICMS can also be passed at multiple different times within a block of trials (Fig. 1A), permitting construction of a timeline of the effects of ICMS-SEF.

\section{Materials and methods}

\section{Subjects and surgical procedures}

Two male rhesus macaque monkeys (Macaca mulatta, monkeys $\mathrm{S}$ and Z) weighing approximately $12-14 \mathrm{~kg}$ performed this experiment. All training, surgical and experimental procedures were approved by the Animal Use Subcommittee of the University of Western Ontario Council on Animal Care, and were conducted in accordance with the Canadian Council on Animal Care policy on the use of laboratory animals, which conforms to the Guidelines laid down by the National Institutes of Health regarding the care and use of animals for experimental procedures. The monkeys' health and weight were monitored daily.

Each animal underwent two surgeries as described previously (Elsley et al., 2007). Briefly, in the first surgery we implanted a head post for head restraint, a scleral search coil for monitoring of twodimensional gaze position and a recording chamber permitting bilateral access to the SEF (stereotactic coordinates Monkey S: AP $=25$, $\mathrm{ML}=3$; Monkey $\mathrm{Z}: \mathrm{AP}=24, \mathrm{ML}=2$ ). In the second surgery, we implanted chronically indwelling bipolar hook electrodes bilaterally in five neck muscles involved in orienting the head both horizontally and vertically. We focus here on the activity of three of these muscles (OCI, obliquus capitis inferior; RCP maj, rectus capitis posterior major; SPL cap, splenius capitis; see Fig. 4), as these muscles form the core of the horizontal head-turning synergy (Corneil et al., 2001) and are robustly recruited by extracellular stimulation of the oculomotor system (Corneil et al., 2002; Elsley et al., 2007; Farshadmanesh et al., 2008; Chapman et al., 2012). Similar profiles of recruitment were observed across all muscles, so for the sake of simplicity we have pooled normalized recruitment levels across all muscles (see below for a description of our normalization procedure).

\section{Intracortical microstimulation}

ICMS-SEF was delivered through tungsten microelectrodes (impedance $0.5-1.2 \mathrm{M} \Omega$ at $1 \mathrm{kHz}$ ) lowered through 23-gauge tubes secured within a Delrin grid. The stimulation sites from which we derived the data reported here are a subset of the sites visited previously (Chapman et al., 2012), screened to be those from which a saccade with a predominantly horizontal component could be evoked (neural activity in the vicinity of the stimulation electrode was not systematically recorded). Briefly, SEF sites were classified as those sites from which a prolonged train of biphasic stimulation pulses $(100 \mu \mathrm{A}, 300 \mathrm{~Hz}, 200 \mathrm{~ms})$ reliably elicited a saccade while a monkey looked around the room; as reported in our previous work stimulation at these parameters also evokes a robust neck muscle response (Chapman et al., 2012). Once an eligible SEF site was encountered, stimulation duration was shortened to $30 \mathrm{~ms}$. Thus, during the behavioral paradigm described below, ICMS-SEF consisted of ten individual stimulation pulses, delivered at $300 \mathrm{~Hz}$ (i.e. $100 \mu \mathrm{A}, 300 \mathrm{~Hz}, 30 \mathrm{~ms}$ ). While stimulation duration was designed to be very short to preclude evoked saccades, the fixed stimulation current of $100 \mu \mathrm{A}$ is considerably higher than that used in some studies of the SEF with longer stimulation durations (Schlag \& Schlag-Rey, 1987; Martinez-Trujillo et al., 2003; Stuphorn \& Schall, 2006), but in the same range as that used in others (Chen \& Walton, 2005; Yang et al., 2008; Kunimatsu \& Tanaka, 2012). Our choice for a fixed stimulation current was based on our previous work in the FEFs (Corneil et al., 2010), where we showed marked differences in saccadic vs. neck electromyographic (EMG) thresholds depending on the size of the characteristic vector. Given this variability, we opted for a fixed stimulation current, and adopted the level used in our previous SEF work (Chapman et al., 2012).

\section{Behavioral paradigm}

Our general experimental setup has been described previously (Chapman et al., 2012). Briefly, the animals were seated in a primate chair with either the head restrained or unrestrained, facing an array of tri-colored (red, green or orange), equiluminant LEDs. The monkeys were trained as described previously (Chapman \& Corneil, 2011) to generate a pro-saccade or an anti-saccade to a peripheral cue depending on the color of a central fixation point (FP; Fig. 1A) for a liquid reward delivered through a head-fixed sipper tube. Trials began with the removal of a diffuse, white background light that prevented dark adaptation. A red or a green FP was then presented directly in front of the monkey. The monkey was required to look at the FP within $1000 \mathrm{~ms}$ and hold gaze within a computercontrolled window (radius of $2.5^{\circ}$ ) for $1250 \mathrm{~ms}$. A red stimulus (the peripheral cue) was then presented randomly to the left or the right of the FP. Cue locations were fixed at either 10,15 or $20^{\circ}$, with the eccentricity chosen to be the closest match to the horizontal component of the saccade evoked with longer-duration SEF stimulation. The monkeys had $1000 \mathrm{~ms}$ to either look toward (if the FP was red) or away (if the FP was green) from the cue, and fixate for a subsequent $600 \mathrm{~ms}$. The radius of acceptance windows around the correct goal location was $40 \%$ of cue eccentricity, to allow for the inaccuracy of anti-saccades in the dark. On anti-saccade trials, an additional green stimulus was illuminated at the correct goal location $300 \mathrm{~ms}$ after the correct anti-saccade as reinforcement. A 1000-ms inter-trial interval was provided between each trial. These behavioral constraints were identical for trials with or without ICMS-SEF.

Pro- and anti-saccade trials were presented with equal probability with replacement for incorrectly performed trials (i.e. trials where the monkeys did not obtain a reward). Short-duration ICMS-SEF was delivered on two-thirds of all trials, with the other trials designated as control trials. On a given stimulation trial, ICMS-SEF was delivered at a single time-point relative to cue presentation $(-1150$, $-817,-483,-150,10,43,77$ or $110 \mathrm{~ms}$, with negative numbers meaning that stimulation preceded cue presentation; Fig. 1A). We define the time preceding cue presentation as the fixation interval, and the time after cue presentation as the post-cue interval. Across a block of 600 correctly performed trials, all potential trial conditions were presented an equal number of times by a customized LABVIEW program that controlled all aspects of the experiment through a PXI controller (National Instruments, Austin, TX, USA) at $1 \mathrm{kHz}$. To ensure that the monkeys encountered all trial types at the appropriate probability throughout the block of trials, they completed subblocks of 48 trials consisting of four repeats of unique control trials (pro- or anti-saccades to the left or right, and hence 16 trials) and one repeat of each unique stimulation condition (pro- or 

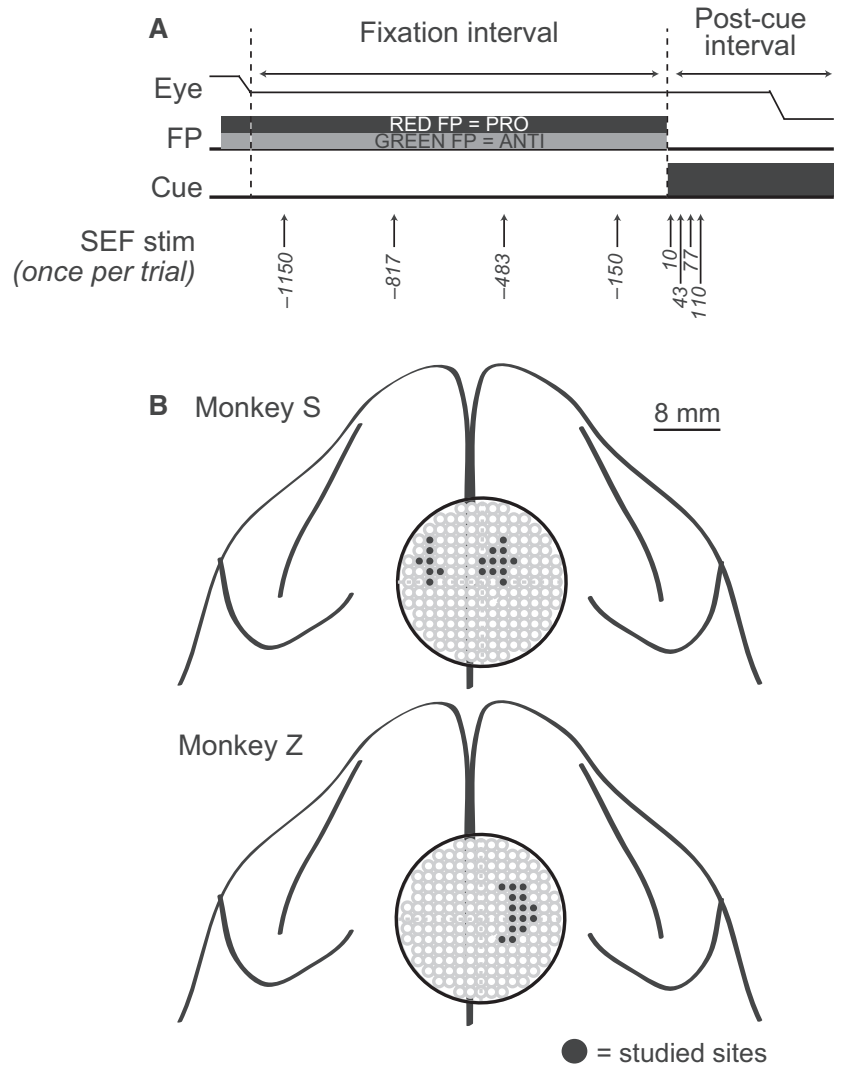

FIG. 1. Behavioral task and depiction of stimulation locations. (A) Monkeys performed an interleaved pro- or anti-saccade task in which the instruction to look either toward or away from a peripheral cue was conveyed by a red or green fixation point (FP), respectively. Short-duration ICMS-SEF was passed on a subset of trials. Although ICMS-SEF was passed only once on any given stimulation trial, the exact time of stimulation relative to cue onset varied between trials. Cue onset divided the fixation interval from the post-cue interval. (B) Representation of guide tube locations from which the data for this paper were derived. These locations are a subset of those studied by Chapman et al. (2012), selected to be those from which predominantly horizontal saccades could be evoked.

anti-saccades to the left or right, over eight possible stimulation times, and hence 32 trials). We stress that ICMS-SEF was only delivered once on a given stimulation trial, but the exact time of stimulation varied amongst eight different possibilities.

\section{Data collection and processing}

During the experiment, we measured the EMG activity of neck muscles as described in detail previously (Elsley et al., 2007). Briefly, multi-motor unit EMG was recorded via bipolar hook electrodes implanted chronically into a given muscle, and subsequently differentially amplified, filtered $(100 \mathrm{~Hz}-4 \mathrm{kHz})$ and digitized at $10 \mathrm{kHz}$. Offline, EMG signals were further subjected to a $60-\mathrm{Hz}$ notch filter, rectified and then bin-integrated in 1-ms bins. We also recorded two-dimensional (2-D) gaze position in space and, when head-unrestrained, 2-D head position in space via a second search coil secured to the head post in the frontal plane. Offline analysis of eye, head, gaze and EMG signals was conducted using customized MATLAB (The Mathworks, Natick, MA, USA) programs. A customized interface permitted trial-by-trial inspection of all trials, and this interface also automatically detected the start and end of saccades and head movements using velocity criteria (30 or $10 \%$, respectively), and classified trials into correct or erroneous pro- or anti-saccades (i.e. an anti-saccade error occurs when the monkey looked incorrectly to the cue on an anti-saccade trial). Trials could be rejected if necessary (e.g. if the monkey neither looked initially to the goal location nor incorrectly to the cue on anti-saccade trials, or if there were excessive levels of EMG activity due to idiosyncratic shifts in posture); such rejections occurred on far fewer than $1 \%$ of all trials (mean \pm SD: $0.11 \pm 0.16 \%$, range $0-0.55 \%$ ).

To facilitate the comparison of EMG recruitment levels across muscles, monkeys and different experimental days, we normalized EMG levels to the level of recruitment attained in the $50 \mathrm{~ms}$ preceding cue onset, pooled across pro- and anti-saccade trials. This normalization procedure was performed on each muscle recording within a given day, and is necessary as each EMG electrode has a unique impedance.

\section{Results}

We acquired a complete block of 600 trials each from a total of 52 unique stimulation locations distributed over the left and right SEF (Fig. 1B; 24 from monkey S, 28 from monkey Z). A subset of this dataset was collected with the head unrestrained (six from monkey $\mathrm{S}$, eight from monkey $\mathrm{Z}$ ). We first examined our data for any systematic effects of restraint, comparing the change in reaction time (RT) or error rates compared with control trials, or the change in evoked neck EMG responses compared with an earlier stimulation interval. The only significant result of this analysis was that neck EMG responses evoked during the post-cue interval tended to be greater with the head unrestrained. Subsequent analyses of data restricted to that obtained with the head restrained revealed the same pattern of results emphasized below, and hence our results are not simply due to the inclusion of head-unrestrained data. We therefore pooled data across head-restrained and head-unrestrained sessions. We also pooled data across stimulation of the right and left SEF, and refer to cue locations, saccades and muscles as being contra- or ipsilateral to the side of SEF stimulation. Our convention is to refer to saccade direction, and hence a correct contralateral anti-saccade requires the monkey to look away from an ipsilateral cue. A contralateral anti-saccade error is one where the monkey saccades incorrectly to a contralateral cue.

We first analysed whether short-duration ICMS-SEF directly evoked saccadic eye movements. During the fixation interval, saccades following stimulation but preceding cue onset occurred on fewer than $1 \%$ of all appropriate stimulation trials. We also found no consistent difference in the change of eye position during the fixation interval between control trials and trials with stimulation during this interval (a $t$-test of the eye position changes reached significance in only three of the 52 sessions, and only one of these sessions showed the contralateral change in eye position that would be expected from stimulation). These analyses show that the animals maintained fixation during short-duration ICMS-SEF. We also found that the proportion of express saccades, which we leniently defined as RTs between 60 and $120 \mathrm{~ms}$, was $2.5 \pm 5.8 \%$ on control trials, and never exceeded $3 \%$ for trials with stimulation delivered at any interval. These analyses emphasize the inability of short-duration ICMS-SEF to directly evoke saccades, even when delivered during the post-cue interval.

\section{Short-duration ICMS-SEF selectively impairs anti-saccade performance}

On control trials, both monkeys generated higher error rates (Fig. 2) and longer RTs (Fig. 3) on anti- vs. pro-saccade trials. Furthermore, 
A

\section{Contralateral errors, re: SEF stimulation}

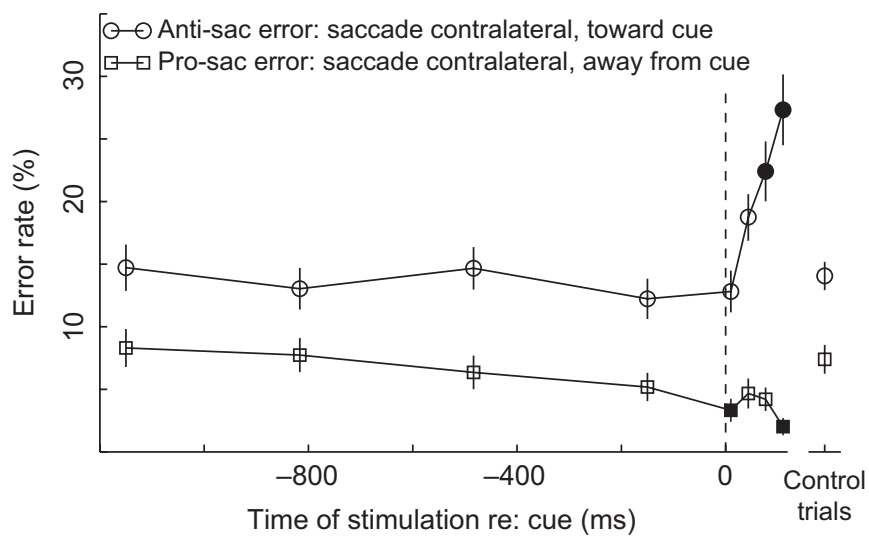

\section{B Ipsilateral errors, re: SEF stimulation}

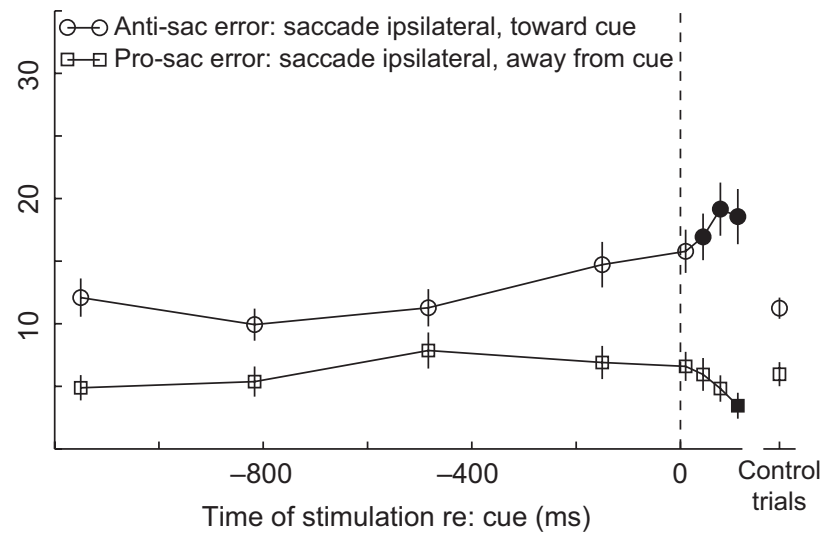

C Change in contralateral error rate, compared to control

D Change in ipsilateral error rate, compared to control
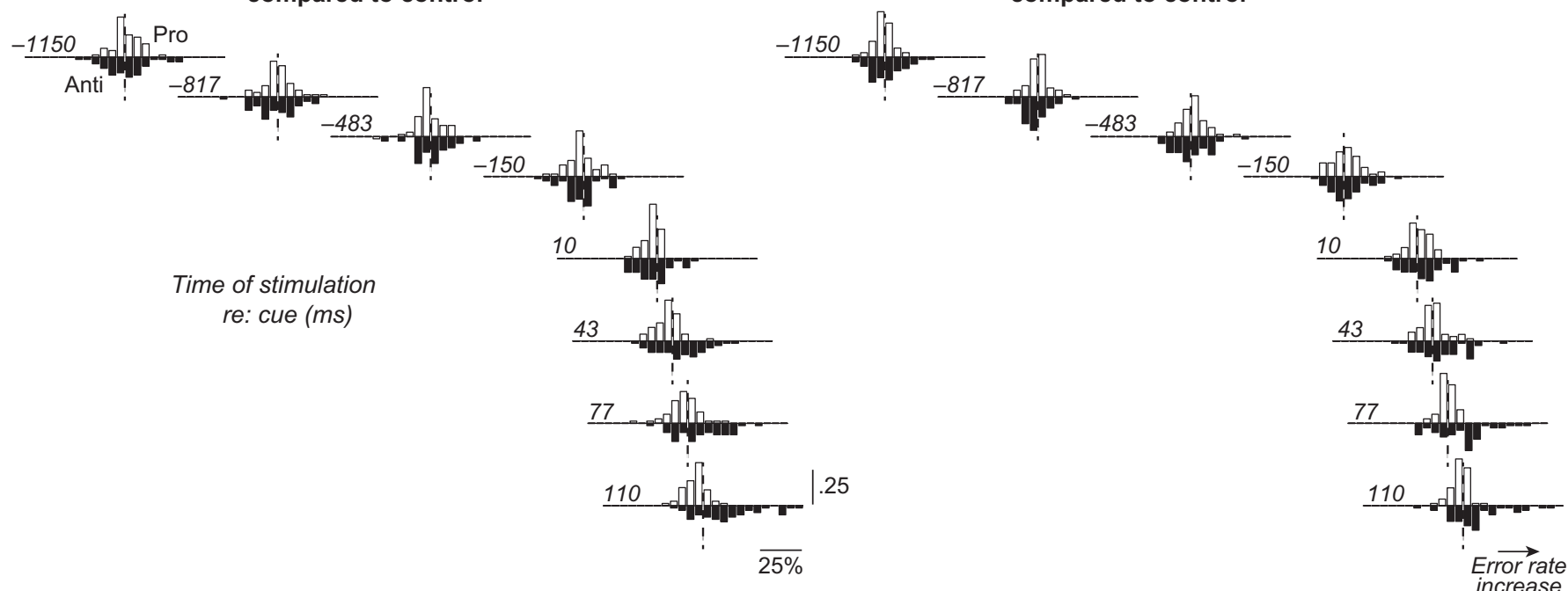

FIG. 2. Error rates plotted as a function of the time of ICMS-SEF relative to cue presentation contralateral (A) or ipsilateral (B) to the stimulating electrode. The data from control trials without stimulation are shown to the right of each subplot. Errors bars show SEM. Filled symbols represent error rates on stimulation trials that were significantly different from the error rates on control trials (paired $t$-test, corrected for multiple comparisons). Frequency histograms in $\mathrm{C}$ and D represent the changes in error rate relative to control trials in a given session for both pro-saccades (upward histograms) and anti-saccade (downward histograms) for each stimulation time-point. Rightward shifts in these histograms relative to the zero point (vertical dashed line) represent increases in error propensity.

the RTs of anti-saccade errors approached the RTs of pro-saccades (Fig. 3), and are not in the range of express saccades. These patterns replicate those reported in previous studies in monkeys generating intermixed pro- and anti-saccades (Amador et al., 1998; Bell et al., 2000).

The influence of short-duration ICMS-SEF on error rates is shown in Fig. 2, collapsed across all experimental sessions. Short-duration ICMS-SEF exerted a negligible influence on either pro- or anti-saccades when delivered during the fixation interval (i.e. to the left of the vertical dashed line), but progressively impacted error rates the later it was delivered during the post-cue interval. This influence depended on whether the monkeys were preparing to generate a pro- or anti-saccade, with ICMS-SEF increasing bilateral error rates on anti-saccade trials, and decreasing bilateral error rates on pro-saccade trials. We did observe an asymmetry in the increase in error rates on anti-saccade trials, with short-duration SEF stimulation causing a larger increase in contralateral (Fig. 2A) vs. ipsilateral anti-saccade errors (Fig. 2B).
A three-way repeated-measures analysis of variance (ANOvA) of error rate across the factors of task (pro- or anti-saccades), direction (contra- or ipsilateral to stimulation) and time of stimulation (including control trials) revealed significant effects of task and time of stimulation $\left(P<10^{-5}\right)$, and significant two-way and three-way interactions between all factors (task and direction: $P=0.02$; task and stimulation time: $P<10^{-5}$; direction and stimulation time: $P=0.003$; task, direction and stimulation time: $P=0.03$ ). Subsequent two-way repeated-measures ANOVAs of error rates on pro- or anti-saccade trials revealed a far greater influence of stimulation time on anti-saccade vs. pro-saccade trials, suggesting that the three-way interaction between task, direction and stimulation is primarily driven by the anti-saccade error rate. The filled symbols in Fig. 2 show data that differed significantly from the respective control trials (paired $t$-tests, Bonferroni-corrected for multiple comparisons), and the frequency histograms in Fig. $2 \mathrm{C}$ and D represent the change in error rate vs. control trials for pro- or anti-saccades for each stimulation interval. The greater impact of ICMS-SEF on anti-saccade error 

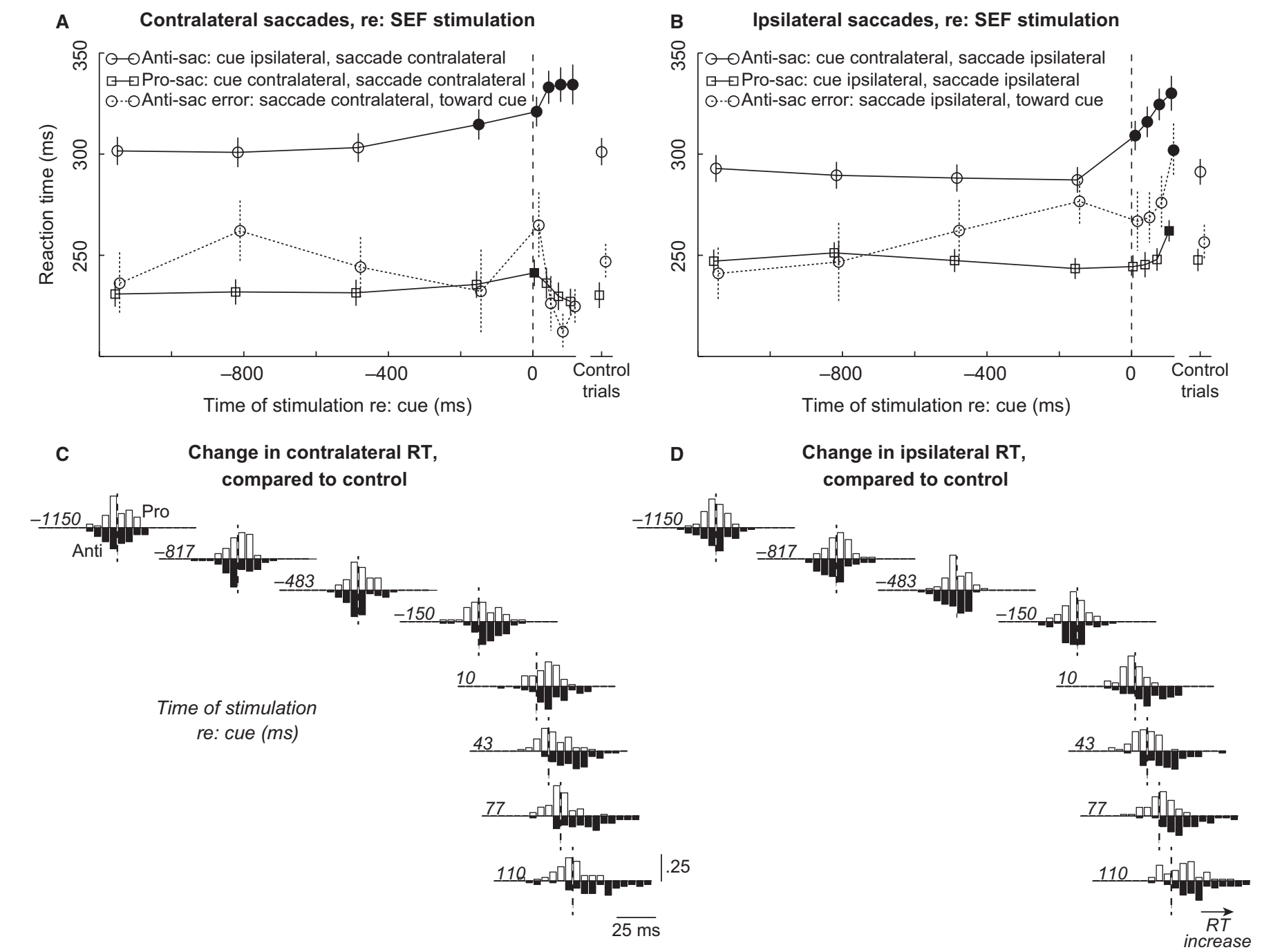

FIG. 3. Saccadic reaction time (RT) plotted as a function of the time of ICMS-SEF relative to cue presentation. The format is as in Fig. 2, with the addition of the RTs for anti-saccade errors toward the peripheral cue. Some of the traces have been shifted slightly on the $x$-axis to permit visualization of the error bars. Frequency histograms represent the change in RT referenced to control trials.

rate across our sample can be appreciated by gauging the degree of shift of these histograms away from zero (rightward shifts convey increases in error rate). Note also that the histograms shifts tend to be greater for contralateral vs. ipsilateral anti-saccade errors for the later stimulation intervals, emphasizing some degree of laterality to the change in anti-saccade error rate.

The influence of short-duration ICMS-SEF on RTs is shown in Fig. 3 in a similar fashion. As with error rates, the influence of ICMS-SEF on correct RTs is highly dependent on the task, and on the timing of stimulation relative to cue presentation (Fig. 3). Shortduration ICMS-SEF during the fixation interval exerted only a minor effect on RTs, but exerted a much greater effect when delivered after cue onset on anti-saccade trials, progressively prolonging the RTs of correctly performed anti-saccades in either direction. Interestingly, short-duration ICMS-SEF had little effect on the RTs of contralateral pro-saccades, although we did observe a modest increase in the RTs for pro-saccades to an ipsilateral cue for later stimulation times. Finally, the RTs of anti-saccade errors displayed a dependency with saccade direction, becoming shorter for errors made to contralateral cues, and longer for errors to ipsilateral cues.
A three-way repeated-measures ANOvA of the RTs of correctly performed saccades revealed significant effects of task and time of stimulation (both $P<10^{-5}$ ), and significant two-way and three-way interactions of all factors (all $P<0.01$ ). Subsequent two-way repeated-measures ANOVAS of error rates on pro- or anti-saccade trials revealed that the three-way interaction was due to a greater influence of cue direction on pro-saccade vs. anti-saccades, and time of stimulation of anti-saccade vs. pro-saccade trials. The filled symbols in Fig. 3A and $\mathrm{B}$ and the histograms in Fig. $3 \mathrm{C}$ and $\mathrm{D}$ give a sense of the consistency in these changes across the sample, and permit a comparison of the magnitude of changes in RT across different tasks and directions. In particular, note the robustness of the increases in bilateral anti-saccade RT for stimulation times in the post-cue interval (increases were observed in the vast majority of sessions).

We also represent the RTs of anti-saccade errors in Fig. 3. The RTs of anti-saccade errors always exceeded $200 \mathrm{~ms}$, even for the latest stimulation time, emphasizing again that ICMS-SEF is neither driving saccades directly nor evoking express saccades. Note also how the RTs for ipsilateral anti-saccade errors are longer than the RTs for ipsilateral pro-saccades for later stimulation times (Fig. 3B). 
This observation is relevant to the potential influence of ICMS-SEF on anti-saccade performance, and will be returned to in the Discussion.

To summarize, short-duration ICMS-SEF influenced both the error rates and the RTs of pro- and anti-saccades. This influence is characterized by strong dependencies with both the task, with error rates and RTs increasing for anti-saccades, and the time of stimulation, with greater influences emerging the later stimulation is passed relative to cue onset. Importantly, the observation of a greater influence of ICMS-SEF on saccades in anti- vs. pro-saccades alleviates concerns about the animals anticipating the delivery of stimulation, given that half of our stimulation times occur after cue onset. If the animals were being distracted by the increasing possibility of ICMSSEF as the trial progressed, such distraction may have been manifest in a similar ways on pro- and anti-saccade trials, which differs from what we observed. Furthermore, although we did observe some asymmetries with saccade direction, short-duration ICMS-SEF increases the error rate and RT of both ipsilaterally and contralaterally directed anti-saccades.

\section{Profile and quantification of neck EMG evoked by short- duration ICMS-SEF}

We now describe the effect of short-duration ICMS-SEF on neck muscle recruitment, focusing first on the recruitment evoked bilaterally on muscles involved in horizontal head turns, and then on how we have quantified such evoked recruitment. The data in Fig. 4A are taken from a single representative session, and show neck muscle recruitment aligned to stimulation onset collapsed across all experimental conditions. As with longer duration ICMS-SEF (Chapman et al., 2012), short-duration ICMS-SEF robustly increased the activity of contralateral turning muscles, and robustly decreased the activity of ipsilateral turning muscles. Such lateralized recruitment is the hallmark of a horizontal head-turning synergy (Corneil et al., 2001), and is seen following stimulation of all oculomotor structures studied to date (Corneil et al., 2002; Elsley et al., 2007; Farshadmanesh et al., 2008; Chapman et al., 2012). Note, however, that the magnitude and exact timing of the recruitment sequence evoked by ICMS of an oculomotor structure does differ from that used volitionally; in particular, the absolute magnitude of agonist recruitment is less for volitional movements, and the recruitment or silencing of a given muscle tends to be more staggered in volitional movements as well (Corneil et al., 2001).

Our quantification of the effects of short-duration ICMS-SEF focuses on the activity of the contralateral muscles, as the strength of inhibition of ipsilateral muscles cannot be quantified and depends on the level of background EMG preceding ICMS-SEF. We emphasize again that ipsilateral muscle inhibition always accompanied contralateral muscle recruitment, consistent with ICMS-SEF recruiting a contralateral head-turning synergy, rather than causing a generalized arousal that would presumably be related to a bilateral increase in both ipsilateral and contralateral muscle tone. As mentioned in the Methods, we pooled normalized EMG activity across the three contralateral muscles, as similar profiles of recruitment were observed on OCI, RCP maj and SPL; such normalized activity is represented in Figs 4-6. We quantified both the baseline level of neck EMG preceding stimulation (averaged over $10 \mathrm{~ms}$ preceding stimulation), and the increase in neck EMG above baseline (see representation of these measures in the top row of Fig. 4A). Our rationale for doing so is because our previous work (Chapman \& Corneil, 2011) detailed modulation of neck muscle activity during the fixation period with the consolidation of the instruction to make a pro- or
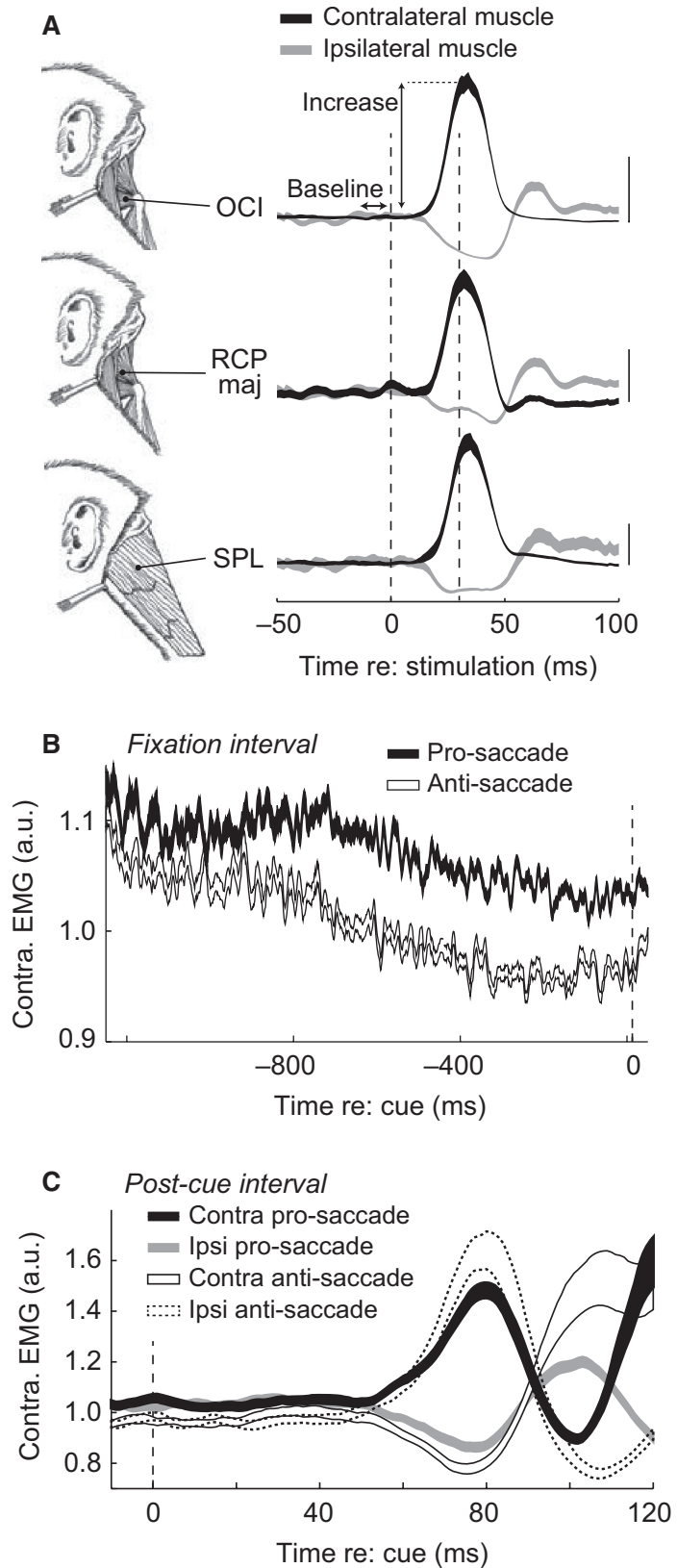

FIG. 4. Neck muscle activity during an interleaved pro- and anti-saccade task. (A) Evoked neck muscle responses were measured bilaterally on three muscles involved in head turning: obliquus capitis inferior $(\mathrm{OCI})$, rectus capitis posterior major (RCP maj) and splenius capitis (SPL). On each of these, short-duration ICMS-SEF robustly increased the contralateral muscle activity, and decreased ipsilateral muscle activity. Scale bars, $10 \mu \mathrm{V}$. The depiction of "baseline" and "increase" in the upper subplot shows the measures represented in Figs 5 and 6 . The background activity on these muscles also changed during the fixation interval $(\mathrm{B}$; attaining greater activity prior to pro- vs. anti-saccades), and during the post-cue interval (C; with a visual response evolving $\sim 75 \mathrm{~ms}$ after ipsilateral cue presentation for both pro- and antisaccades). EMG data in $\mathrm{B}$ and $\mathrm{C}$ are normalized to the level of activity in the $50 \mathrm{~ms}$ preceding cue onset, pooled across pro- and anti-saccade trials.

anti-saccade, and during the post-cue interval depending on the side of the cue. We summarize these patterns briefly here as they influence the interpretation of the neck EMG evoked by ICMS-SEF.

On control trials, neck EMG during the fixation interval began to diverge gradually $\sim 300-400 \mathrm{~ms}$ after acquisition of the FP (Fig. 4B), eventually becoming $\sim 10 \%$ higher prior to cue onset in 


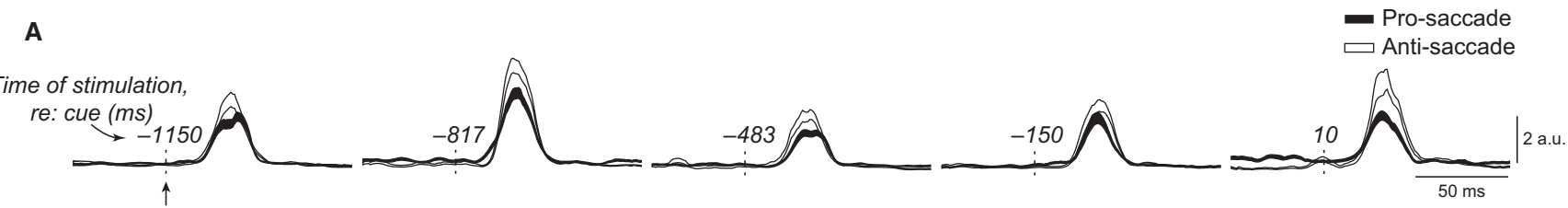

Onset of ICMS-SEF

B

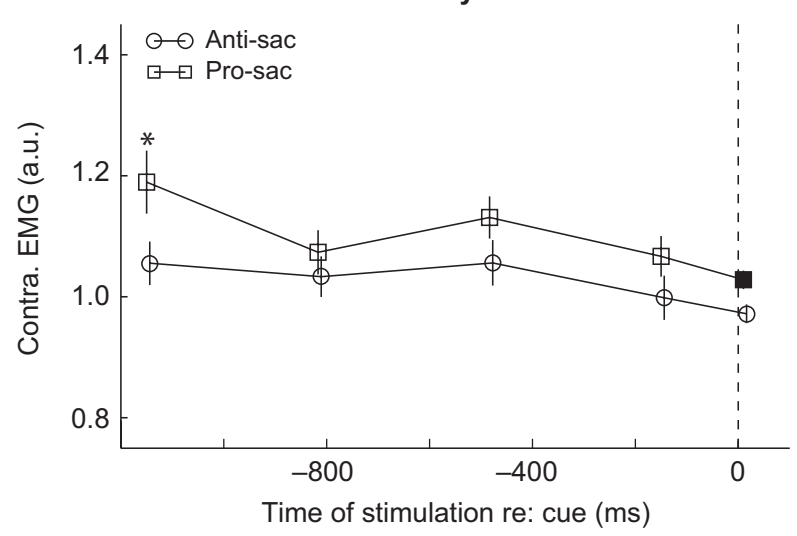

D

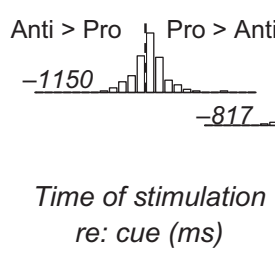

Comparison of baseline EMG

(Pro - Anti)
C EMG increase above baseline

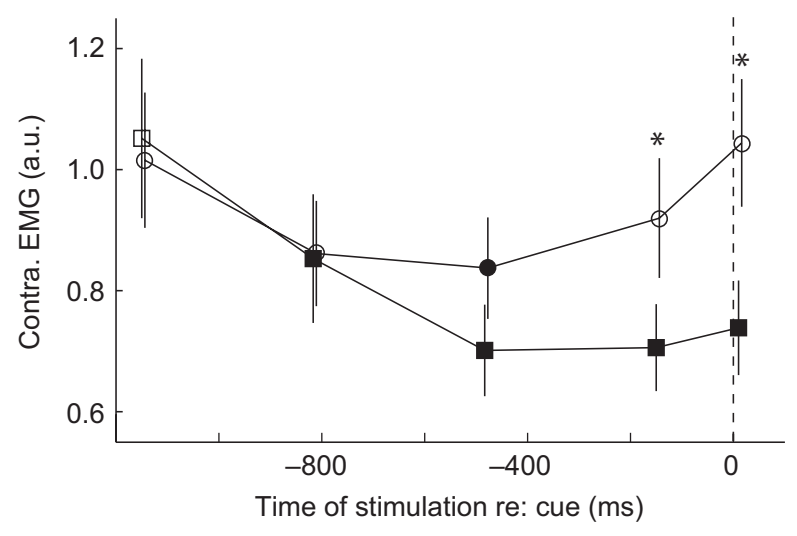

E Comparison of EMG increase above baseline (Pro - Anti)

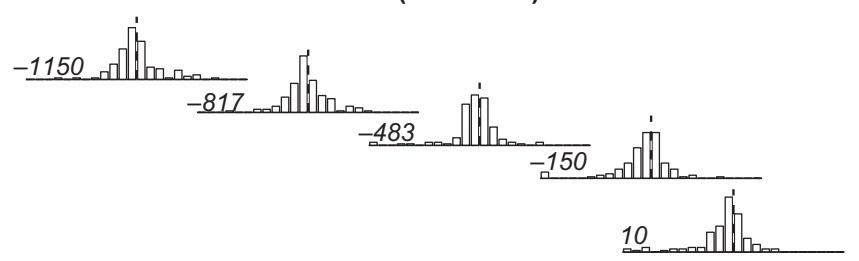

FIG. 5. Evoked neck muscle responses during the fixation interval. (A) Normalized neck muscle traces from contralateral muscles from a representative site (the same as shown in Fig. 4A), showing recruitment from $50 \mathrm{~ms}$ before to $100 \mathrm{~ms}$ after ICMS-SEF (dashed vertical line), segregated by the time of ICMSSEF relative to cue onset. Data are segregated by task, showing recruitment prior to the generation of pro-saccades (solid contours) or anti-saccades (empty contours); contours represent the area subtending the mean \pm SE. (B-E) Quantification of results across the sample, showing the background EMG activity immediately preceding ICMS-SEF (B), and the magnitude of the evoked neck muscle response above baseline (C). The format is as in Fig. 2, except that filled symbols represent significant differences compared with the first stimulation interval (paired $t$-tests, corrected for multiple comparisons), and an asterisk $(*)$ represents significant differences between data from pro- and anti-saccade trials at a given stimulation time. Frequency histograms in D and E represent the difference in a given measure on pro- vs. anti-saccades for a given time of stimulation, with leftward shifts from zero denoting sessions where a greater magnitude was measured on anti-saccade trials.

pro- vs. anti-saccade trials. Such divergence reflects a top-down consolidation of task instruction, and was observed in both monkeys $\mathrm{S}$ and Z. This pattern of recruitment was seen in one of two different monkeys in our previous study (Chapman \& Corneil, 2011), with the other monkey displaying significantly greater activity before anti-saccades. The gradual decrease in neck EMG activity during the fixation interval also shows that the animals were not cocontracting their neck, as might have been expected if they were bracing for the increasing probability of stimulation as the fixation interval wore on. During the post-cue interval, neck EMG further diverged abruptly $\sim 60-70 \mathrm{~ms}$ after cue onset, increasing following the onset of cues on the same side as the recorded muscle, and decreasing following the onset of cues on the opposite side. This is the visual response on neck muscles that we have reported previously in a variety of tasks (Corneil et al., 2004, 2008; Chapman \& Corneil, 2011); relative to the side of the SEF electrode, contralateral muscles increase following the presentation of contralateral cues and decrease following the presentation of ipsilateral cues, regardless of whether the monkey ultimately looks toward or away from the cue. Following this visual response, we observed a rebound in recruitment that peaked about $90-110 \mathrm{~ms}$ after cue presentation, with activity decreasing following contralateral cues, and increasing following ipsilateral cues.

\section{Progressively greater evoked neck EMG responses emerge on anti-saccade trials during the fixation interval}

We now turn to the quantification of the EMG response evoked by short-duration ICMS-SEF. We focus first on the activity evoked during the fixation interval, collapsed across saccade direction. We include the first stimulation time in the post-cue interval (i.e. $10 \mathrm{~ms}$ after cue presentation), as this precedes the arrival of visual information in the SEF. Figure 5A displays the normalized EMG response to short-duration ICMS-SEF for a representative site (the same as shown in Fig. 4A), segregated by task and the time of stimulation relative to cue onset. ICMS-SEF evoked robust recruitment at all 
A

Contra pro-saccade - Ipsi pro-saccade $\square$ Contra anti-saccade ::.:: Ipsi anti-saccade
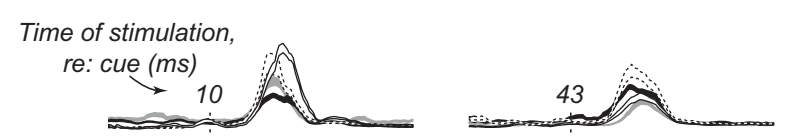

B

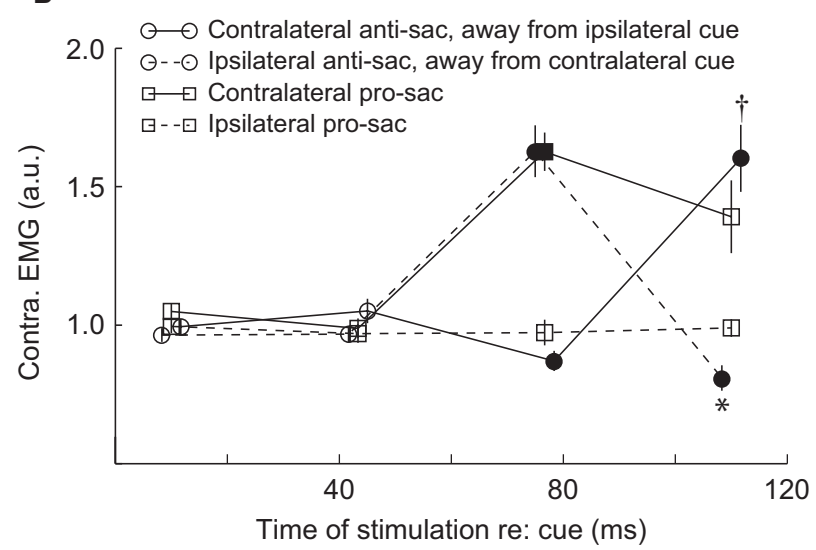

D

Comparison of baseline EMG

(Contra - Ipsi)

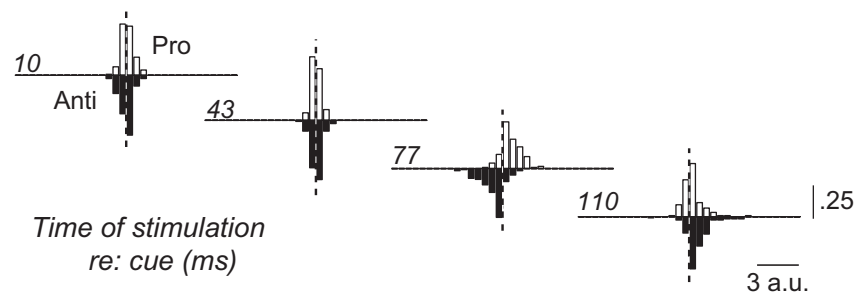

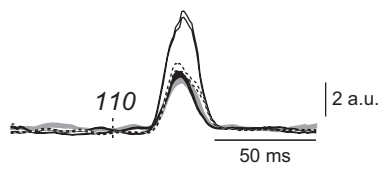

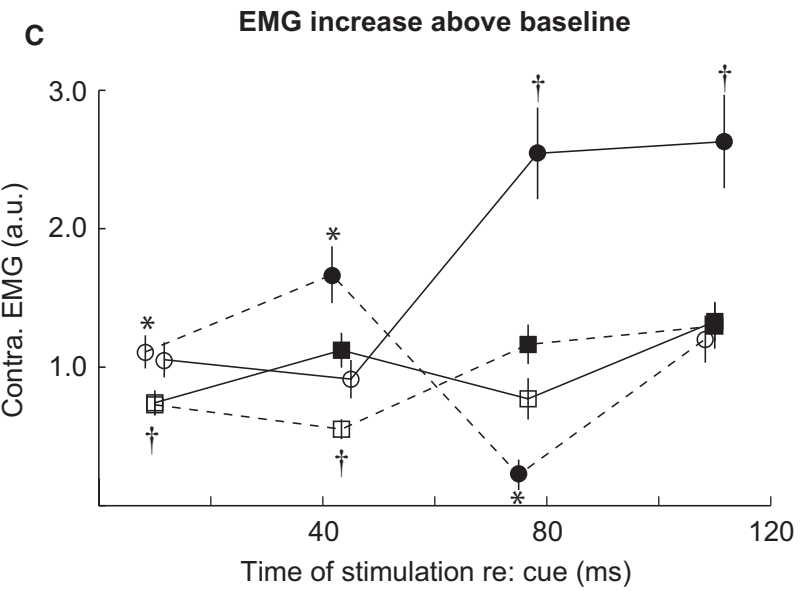

E above baseline (Contra - Ipsi)

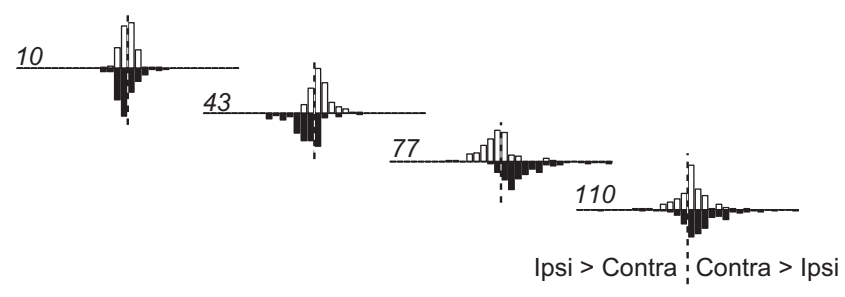

FIG. 6. Evoked neck muscle responses during the post-cue interval. The format is as in Fig. 5, except that data are also subdivided by the side of cue presentation relative to the stimulating electrode. (A) Normalized contralateral neck muscle traces from the representative site. Filled symbols in B and C show results of statistical comparison done to the data at the first stimulation interval for the same condition (paired $t$-tests, corrected for multiple comparisons). ${ }^{*} \dagger$ Significantly different results for evoked responses from pro- vs. anti-saccade trials with the cue on the same side at a given stimulation time (*contralateral pro-saccades vs. ipsilateral anti-saccades; †ipsilateral pro-saccades vs. contralateral anti-saccades; paired $t$-tests, corrected for multiple comparisons). Frequency histograms in $\mathrm{D}$ and $\mathrm{E}$ represent the dependency of the effect on saccade direction, for either pro-saccades (upper histograms) or anti-saccades (lower histograms). Note in general how the lower histograms in E are shifted further from zero than the upper histograms, emphasizing a greater degree of modulation of the stimulation-evoked effect with saccade direction on anti-saccades.

times, but the magnitude of such recruitment depended on both the task and the time of stimulation, with ICMS-SEF evoking the greatest recruitment when delivered just after cue onset in the anti-saccade task. Our analysis of these patterns across our sample is shown in Fig. 5B-E. As shown in Fig. 5C, the increase in evoked neck EMG above baseline diverged progressively as the monkeys prepared to make anti- vs. pro-saccades. Importantly, the magnitude of evoked neck EMG is not simply the reflection of baseline activity (Fig. 5B); ICMS-SEF evoked greater neck EMG as the monkeys prepared to make anti-saccades, despite a lower amount of baseline recruitment preceding stimulation. We observed this trend regardless of eventual saccade direction, and hence the influence of task on stimulation-evoked responses in this interval is not simply an interaction with the subsequent visual response on neck muscles. A repeated-measures two-way ANOvA of the increase in evoked neck EMG above baseline revealed significant effects of task $\left(P<10^{-5}\right)$, time of stimulation $(P=0.0001)$ and the interaction between these two factors $(P=0.007)$. The filled symbols in Fig. $5 \mathrm{~B}$ and $\mathrm{C}$ represent observations that differed significantly (Bonferroni-corrected for multiple comparisons) from that observed at the first stimulation interval prior to the consolidation of task instruction. The histograms in Fig. 5D and E represent the comparison of the baseline or increase above baseline on pro- vs. anti-saccades at each stimulation interval across the sample. Note how the bottom two histograms in Fig. 5E are shifted leftward, meaning that EMG increases above baseline tended to be greater on anti- vs. pro-saccade trials.

While Fig. 5C and E represents the increase in neck EMG above baseline, the absolute level of evoked neck EMG was also greater on anti-saccade vs. pro-saccade trials (data not shown, but note how the divergence in Fig. 5C for the last two stimulation intervals exceeds the divergence in baseline activity). This observation means that ICMS-SEF is not simply driving the muscles to the maximal level of recruitment. Further, note how these EMG increases are much smaller in magnitude than the visual response on neck muscles shown in Fig. 4C, which itself tends to be far less than the neck muscle recruitment that accompanies saccade generation, even when 
head-restrained (Corneil et al., 2004, 2008; Chapman \& Corneil, 2011).

\section{Task- and direction-dependent effects interact on evoked neck EMG responses during the post-cue interval}

Finally, we analysed the neck EMG responses evoked by ICMSSEF delivered in the post-cue interval. These data are further segregated by saccade direction relative to the side of the stimulating electrode, as the evoked neck EMG interacts with the visual response on neck muscles for later stimulation times. Accordingly, we describe the effects of ICMS-SEF at each of the four post-cue intervals in sequence, in reference to the data shown in Fig. 6. Again, Fig. 6 shows data from the representative site (Fig. 6A), and across our sample (Fig. 6B-E).

As mentioned above, the response evoked by SEF stimulation at the earliest post-cue interval (i.e. $10 \mathrm{~ms}$ after cue presentation) precedes the visual response on neck muscles. Accordingly, the increase in EMG activity above baseline depended only on task (being greater on anti-saccades), but not on saccade direction (leftmost traces in Fig. 6A; leftmost series of datapoints, Fig. 6C).

In contrast, the response evoked by SEF stimulation delivered slightly later (i.e. $43 \mathrm{~ms}$ after cue presentation) displayed a marked dependency with both task and saccade direction. At this interval, ICMS-SEF before ipsilaterally directed anti-saccades (dashed lines around empty traces in Fig. 6A; dashed line connecting circles in Fig. 6C) evoked the largest response, followed by stimulation preceding contralaterally directed pro-saccades (solid traces in Fig. 6A; solid line connecting squares in Fig. 6C). Note that both such trials feature cue presentation on the side of the muscle (i.e. contralateral to the side of the stimulating electrode), and hence the evoked response is interacting with the ongoing visual response on neck muscles. Even here, it is clear that the stimulation-evoked effect is greater on anti- vs. pro-saccades, and the consistency of this effect is demonstrated by the shifts in the frequency histograms in Fig. 6E, which represent the difference in saccade direction for either pro(upward histrograms) or anti-saccades (downward histograms). Note in particular the greater shift in the downward histogram, meaning that a greater dependency on saccade direction is observed on antisaccade trials.

Following this, the interaction between cue side and task reverses (for $77 \mathrm{~ms}$ after cue presentation), with the greatest evoked responses preceding contralaterally directed anti-saccades (solid lines around empty traces in Fig. 6A; solid lines connecting circles in Fig. 6C). Here, the interaction is with the evoked neck muscle response and the rebound of activity following the visual response on neck muscles (hence the greatest activity with all trials involving presentation of an ipsilateral cue; i.e. ipsilateral pro-saccades, or contralateral anti-saccades). Even here there is still a dependency on task, as a far greater degree of divergence occurs between ipsilateral and contralateral cues for anti-saccades than for pro-saccades (e.g. compare divergence of circles for anti-saccades vs. squares for pro-saccades; see also the shifts in the frequency histograms for the second last stimulation intervals in Fig. 6E). Across our sample, a similar albeit smaller level of divergence between ipsilateral and contralateral cues for anti-saccades than pro-saccades persisted for the latest stimulation time tested (i.e. rightmost series of data in Fig. 6C).

We analysed the increase in evoked neck EMG above baseline with a repeated-measures three-way ANOVA, and revealed significant effects of task, saccade direction and time of stimulation (all $P<10^{-5}$ ), two-way interactions between task and saccade direction and saccade direction and time of stimulation (both $P<10^{-5}$ ) and three-way interactions between all factors $\left(P<10^{-5}\right)$. The symbols in Fig. $6 \mathrm{~B}$ and $\mathrm{C}$, and the frequency histograms in Fig. 6D and $\mathrm{E}$, represent the significance of various changes, and their significance across the sample.

In summary, while the evoked responses during the post-cue interval interacted with the visual response on neck muscles elicited in response to cue presentation, greater interactions occurred when short-duration ICMS-SEF was passed in the context of anti-saccades rather than pro-saccades. Again, ICMS-SEF is not simply driving neck recruitment to the same absolute level, but is evoking larger overall response on anti-saccades vs. pro-saccades (to appreciate this, compare the divergence between lines in Fig. $6 \mathrm{C}$ vs. B; note as well the different scaling of the $y$-axis).

\section{Discussion}

We delivered short-duration ICMS-SEF while monkeys performed an interleaved pro/anti-saccade task. Consistent with results showing greater SEF activity prior to anti-saccades (Amador et al., 2004), we observed progressively larger effects when stimulation preceded anti-saccades. These effects were diverse and varied in directionality: ICMS-SEF selectively disrupted anti-saccade performance by increasing error rate and prolonging the RTs of correct antisaccades, but also elicited greater recruitment of a contralateral head-turning synergy on anti-saccade trials. From the diversity of our results across both oculomotor performance (error rates and RTs) and neck muscle recruitment emerges a more complete picture of the influence of short-duration SEF stimulation on the oculomotor network. In what follows, we consider what our results say about the functionality of the SEF, and about the application of ICMS in cognitive neuroscience.

\section{Comparison with other results from the SEF}

We consider first the effects of ICMS-SEF on error rates and RTs. One of the most prominent effects of ICMS-SEF is to greatly increase the propensity of anti-saccade errors made toward a contralateral cue (relative to the stimulating electrode; Fig. 2A). While ICMS-SEF also decreased the propensity of pro-saccade errors made away from a contralateral cue (Fig. 2B), it is doing more that simply promoting the generation of a contralateral saccade: ICMS-SEF also increased substantially the propensity of anti-saccade errors made toward an ipsilateral cue (Fig. 2B, although this was less than the increase in propensity for contalateral anti-saccade errors), and decreased the propensity of pro-saccade errors made away from an ipsilateral cue (Fig. 2A). These changes in error propensity cannot be attributed to decreased RTs, as might have been expected from a speed-accuracy tradeoff. Instead, the marked increase in anti-saccade errors accompanied substantial increases in RTs, regardless of direction (Fig. 3). We observed a more subtle and much smaller lateralized effect of SEF stimulation on pro-saccade RTs, with RTs increasing or decreasing for ipsilateral or contralateral pro-saccades, respectively. This latter result resembles that reported previously (Yang et al., 2008).

One plausible explanation of our results is that ICMS-SEF selectively disrupts the animal's ability to generate an anti-saccade, regardless of whether the animal was initially instructed to make a pro- or anti-saccade. This disruption is somewhat lateralized, given the greater increase in propensity for contralateral vs. ipsilateral antisaccade errors, but clearly effects anti-saccades in both directions. Exactly how such disruption occurs remains to be determined, but it 
could be that short-duration ICMS-SEF suppresses subsequent activity in the SEF that is required for anti-saccade generation, or perhaps resets the SEF back to the state adopted at the start of the trial. While this type of mechanism would also have to produce the pattern of neck EMG responses we observed (see below), it would explain the bilateral increase in anti-saccade errors, the bilateral decrease in pro-saccade errors and the bilateral increase in the RTs of correct anti-saccades. We favor this interpretation over an alternative explanation that SEF stimulation favors the production of pro-saccades, given the greater level of SEF activity on anti- vs. pro-saccades (Amador et al., 2004), and because a simple bias toward pro-saccades fails to explain the longer RTs for ipsilateral anti-saccade errors compared with ipsilateral pro-saccades.

Whatever the mechanism, our data show that the influence of ICMS-SEF on anti-saccade performance is also highly time-sensitive, with far greater effects being observed the closer stimulation gets to saccade initiation. This time-sensitivity could arise because the SEF's functionality is only required in the immediate peri-saccadic interval, or because the SEF can recover from disruptive effects of ICMS delivered earlier in the fixation interval. The observed time-sensitivity follows a time-course similar to the evolution of SEF activity during an intermixed pro- and anti-saccade task (Amador et al., 2004), consistent with the functional relevance of this area for anti-saccade performance in the primate.

To our knowledge, previous studies employing ICMS-SEF have not investigated the anti-saccade task, hindering direct comparison of our data with the literature. The effects we report of ICMS-SEF on anti-saccade performance are particularly interesting in light of the report by Stuphorn \& Schall (2006) that subthreshold ICMSSEF improved performance in a stop-signal task by delaying saccade generation. In their case, ICMS-SEF served an adaptive purpose when executive control was occasionally required: by delaying saccades, more time was provided for saccade cancellation. A different picture emerges from our data, where the increase in anti-saccade errors is accompanied by a marked bilateral increase in the RTs of correct anti-saccades (Fig. 3). Although short-duration ICMS-SEF delayed anti-saccades, it did not confer any benefit to anti-saccade accuracy but instead also incurred a substantial cost. The differences between our results and those of Stuphorn \& Schall (2006) could arise from the nature of the behavioral tasks and the state of the oculomotor system at the time of stimulation; in our task monkeys were prepared for an anti-saccade by the time ICMS-SEF exerted the largest effects, whereas saccade cancellation in the stopsignal task is required on a minority of trials. Alternatively (or perhaps additionally), the differences may arise from the parameters of subthreshold stimulation; we opted for shorter durations and higher currents $(30 \mathrm{~ms}$ of $100 \mu \mathrm{A}$ at $300 \mathrm{~Hz}$ ), whereas Stuphorn and Schall used longer durations and lower currents $(200 \mathrm{~ms}$ of $10 \mu \mathrm{A}$ or less at $333 \mathrm{~Hz}$ ). What our results share in common with those of Stuphorn and Schall is the state-dependency; they noted that ICMS-SEF delayed saccades when delivered during a stop-signal task, but expedited visually guided saccades otherwise. In our case, the disruption of performance is greater for anti-saccades, with ICMS-SEF only weakly influencing pro-saccades.

A greater influence of ICMS-SEF on more cognitively demanding tasks is also consistent with the results of Kunimatsu \& Tanaka (2012), who showed greater delays from ICMS-SEF for self-initiated vs. conventional memory-guided saccades. These authors also proposed a mechanism by which ICMS-SEF could either shorten or delay saccade initiation depending on the state of oculomotor preparation at the time of ICMS-SEF. Such a mechanism, wherein the oculomotor system was first pushed closer to saccade threshold by

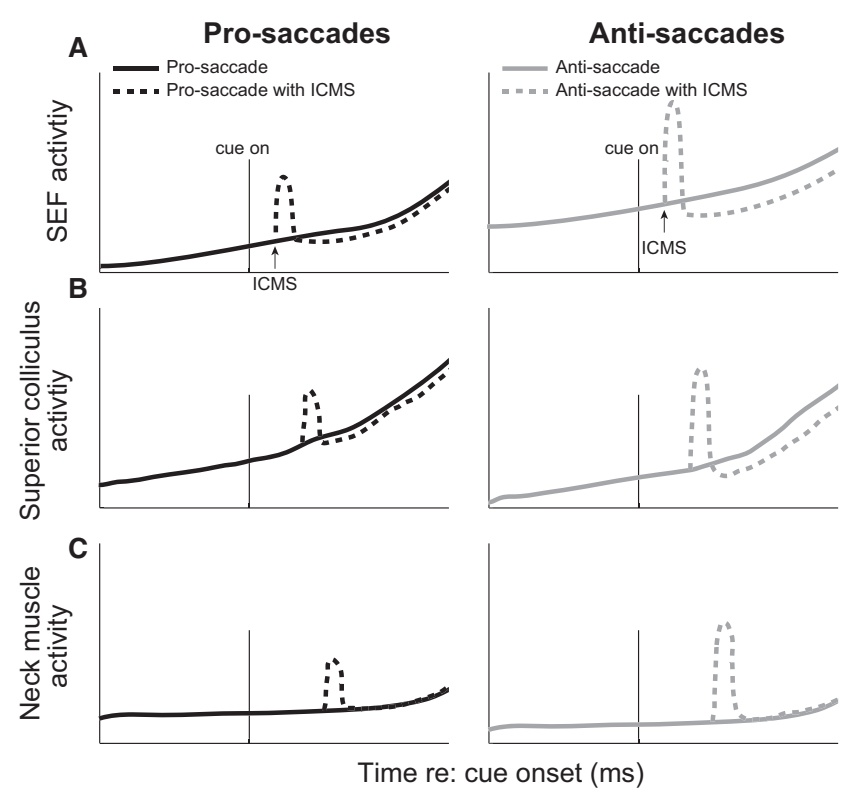

FIG. 7. Schematic representation of a mechanism to explain the multiplicity of the effects of ICMS-SEF. Depicted are the effects of ICMS-SEF delivered just after cue onset in the context of preparing for a pro-saccade (left column) or anti-saccade (right column). As SEF activity is higher prior to antisaccades (A), ICMS triggers a greater amount of activity (dashed lines). Such ICMS-triggered activity courses through downstream oculomotor areas in a feedforward fashion (represented here as the SC; B), culminating in a larger level of neck muscle EMG on anti- vs. pro-saccades (C). After the cessation of ICMS-SEF, there is a greater degree of inhibition or suppression of the SEF and SC on anti-saccades, increasing RTs.

ICMS-SEF but was then temporarily inhibited by post-excitatory suppression, was invoked to explain the variable influence of ICMSSEF on saccade timing. Below, we expand on this concept of a multiphasic effect of ICMS-SEF that includes both an initial excitatory response followed by a subsequent post-excitatory suppression (see Fig. 7). One of the more interesting aspects of our results is that the initial excitatory response to ICMS-SEF can carry a direct motor correlate at the neck.

\section{Comparison with other results outside of the SEF}

To our knowledge, no other study employing ICMS of the oculomotor system during intermixed pro- and anti-saccades has produced the profile of results that we observed from the SEF. For example, the bilateral increases in anti-saccade RTs and error rates from the SEF differ from the largely unilateral increases in RT and error rate observed with stimulation of the dorso-lateral prefrontal cortex (DLPFC) (Wegener et al., 2008), and from the bilateral decreases in the RTs of anti-saccades with negligible changes in error rates observed with stimulation of the anterior cingulate cortex (ACC) (Phillips et al., 2011). What we observed using ICMS-SEF also differs from that produced by stimulation of the caudate nucleus, which produces a greater increase in the RT of contralateral pro-saccades compared with contralateral anti-saccades (Watanabe \& Munoz, 2010). Other work by this group also demonstrated the importance of the exact time of stimulation, with caudate stimulation delivered slightly earlier sometimes shortening RTs (Watanabe \& Munoz, 2011), as well as the importance of the behavioral context at the time of stimulation, with caudate stimulation producing opposite effects depending on whether it was delivered during a behavioral task or not (Watanabe \& Munoz, 2013). While the studies in the 
ACC, DLPFC and caudate nucleus used interleaved pro- and antisaccades as we did, they employed much longer stimulation train durations. Although future studies would ideally use similar stimulation parameters, we can tentatively conclude that the SEF is playing a different role in anti-saccade behavior compared with the ACC, DLPFC or caudate nucleus. What remains to be determined is whether ICMS in these other areas can evoke the multiplicity of effects that we observed in the SEF; such observations would advance the mechanistic interpretation of how ICMS is interacting both with endogenous activity at the time of stimulation and throughout the oculomotor network.

Our use of short-duration ICMS-SEF parallels the use of TMS over the human SEF; both forms of stimulation are short enough to enable delivery at different intervals to construct a timeline of the influence of stimulation on task performance. Single pulses of TMS of the FEFs or DLPFC in humans are also reported to selectively increase the RT and/or error rate of ipsilateral anti-saccades when passed within a critical time window (Muri et al., 1991; Olk et al., 2006; Nyffeler et al., 2007), and bilateral increases in anti-saccade RT and error rates following TMS-FEF have also been reported (Terao et al., 1998). The study by Terao and colleagues also delivered TMS over the SEF in humans, and surprisingly did not observe any significant influence on anti-saccade behaviour. Whether the difference between our results and those in the human TMS literature arise from differences in the species, form of stimulation or exact behavioral paradigm is unclear. TMS can be delivered to monkeys performing oculomotor tasks (Gerits et al., 2011; Valero-Cabre et al., 2012), and hence it should be possible to have direct comparison of different forms of stimulation on anti-saccade behavior in the same species.

Returning to the monkey, our behavioral results resemble those produced following pharmacological inactivation of the ventroanterior and ventrolateral nuclei of the thalamus during an intermixed pro-/anti-saccade task (Kunimatsu \& Tanaka, 2010). Neural activity within these nuclei is consistently greater on anti- than on prosaccade trials, which resembles that reported in the SEF but differs from other frontal and brainstem structures (reviewed by Johnston \& Everling, 2008). Based on this similarity, Kunimatsu \& Tanaka (2010) hypothesized that thalamocortical pathways play an essential role in anti-saccade control. Our results are consistent with this view if one assumes that short-duration ICMS-SEF transiently disrupts processing in this pathway. We are not suggesting that ICMS-SEF selectively disrupts cortico-thalamic processing without influencing other pathways, but speculate that it is this pathway that is primarily responsible for the surprisingly bilateral influences of ICMS-SEF on anti-saccade behavior. The SEF is also richly interconnected with numerous other cortical and subcortical oculomotor structures (e.g. the FEF, ACC, PFC, the superior colliculus (SC), and oculomotor brainstem; reviewed by Johnston \& Everling, 2011), and the effect of ICMS-SEF on these pathways may explain some of the lateralized tendencies in our behavioral results.

\section{Recruitment of a head-turning synergy and interpretation of stimulation-induced effects}

Up to now, we have focused on the impact of ICMS-SEF on antisaccade behavior, which we speculate may arise from an influence on signaling within cortico-thalamic networks. The second major series of results is the augmented recruitment of a contralateral head-turning synergy that accompanies the selective disruption of anti-saccade behavior. During the fixation interval, the magnitude of contralateral muscle recruitment gradually diverged to become larger prior to anti- vs. pro-saccades. Critically, the magnitude of the evoked response did not simply mirror neck muscle recruitment preceding ICMS-SEF. Hence, a straightforward gain of the evoked response that is proportional to motoneuron excitability cannot explain the larger evoked responses as subjects prepare to generate anti-saccades. During the post-cue interval, there is a complex evolution of neck EMG activity that depends on the exact temporal overlap between the evoked EMG response and the visual response on neck muscles. While the baseline EMG activity can explain a portion of the response differential on pro- vs. anti-saccade in this timeframe (e.g. the last three stimulation points), our data show that a larger degree of interaction persists on anti-saccade vs. pro-saccade trials (histograms in Figs 5 and 6). How then can we reconcile larger evoked neck muscle responses on anti-saccade trials with the accompanying disruptions of anti-saccade performance?

We begin by first considering the latency of the neck muscle response evoked by ICMS-SEF. As shown in Fig. 4A, the latency of the evoked neck muscle response is very short, beginning 25$30 \mathrm{~ms}$ after stimulation onset and peaking after the stimulation train. We have previously quantified neck muscle response latencies to ICMS-SEF using a variety of methods to be in the range of $30 \mathrm{~ms}$, leading any evoked saccades by $\sim 40-70 \mathrm{~ms}$ on average (Chapman et al., 2012). The large difference between the onset latencies of neck muscle responses vs. saccades permits the use of short-duration stimulation as a probe of the excitability of the oculomotor system (Corneil et al., 2007). The short latency of the evoked neck muscle response implicates a largely feedforward mechanism from the frontal cortex, through the oculomotor brainstem, and from there to spinal cord and motor periphery. The SEF is connected to a number of oculomotor areas within the brainstem, including the intermediate layers of the SC and other oculomotor structures in the pontomedullary reticular formation; either of these could serve as intermediary relays between the SEF and spinal cord [see Chapman et al. (2012) for more detailed considerations]. It is also possible that the SEF's influence over neck muscle recruitment is mediated through the FEF, as neck muscle response latencies from this structure are $\sim 5$ $10 \mathrm{~ms}$ shorter than from the SEF (Elsley et al., 2007). Regardless of the precise cortical route, the greater responsiveness of the cephalovs. oculomotor circuits is consistent with a series of results in humans and monkeys showing correlates of imposed or adopted subthreshold oculomotor plans in the motor periphery at the neck (Corneil et al., 2004, 2008; Rezvani \& Corneil, 2008; Goonetilleke et al., 2010, 2011). We (Corneil, 2011) and others (Galiana \& Guitton, 1992; Pélisson et al., 2001; Gandhi \& Sparks, 2007) have emphasized the potential role of the omni-pause neurons in the brainstem, which appear to selectively inhibit premotor oculomotor circuits for saccadic gaze shifts without imparting a similar level of influence on cephalomotor commands.

Our results also have implications for a potential role of the SEF in eye-head coordination. A central question in motor coordination is how the brain selects a particular pattern of multisegmental coordination from a limitless space of solutions that could all achieve a desired goal (Bernstein, 1967). This question applies to head-unrestrained gaze shifts, as a given isometric gaze shift can be brought about by varying contributions of the eyes in the head, and head on body. Contextual coordination of the eyes and head is readily observed in both humans and monkeys (e.g. Oommen et al., 2004; Monteon et al., 2012), and recent neurophysiological results have detailed a potential role for the FEF in contextual coupling of the eyes and head (Knight, 2012; Monteon et al., 2012). Our observations that neck muscle responses evoked by ICMS-SEF also vary with context (see also Chen \& Walton, 2005), in this case with the 
instruction to prepare for a pro- or anti-saccade, is consistent with the possibility that the SEF may also provide a substrate for the flexible implementation of strategic contexts with oculomotor plans.

\section{A mechanism for ICMS-SEF's effects on anti-saccade behavior and neck muscle recruitment}

How can we explain the seemingly paradoxical effects of ICMSSEF on anti-saccade behavior and neck muscle recruitment? We speculate that our findings arise from both feedforward and feedback influences of ICMS-SEF throughout the oculomotor system. We illustrate our speculations in Fig. 7 by showing plausible activity profiles within the SEF, the SC (as an intermediary oculomotor area downstream from the SEF) and at the neck. Our speculative mechanism is an extension of that proposed by Kunimatsu \& Tanaka (2012), with added considerations of the comparative effect of consolidation of task instruction to make a pro- or anti-saccade task, and activity profiles at the downstream SC and neck. For this example, ICMS-SEF is delivered shortly after cue onset, before the arrival of visual information.

SEF activity is higher on anti- vs. pro-saccade trials at the time of ICMS-SEF (Schlag-Rey et al., 1997; Amador et al., 2004). Accordingly, we assume that greater amounts of activity are evoked in the SEF, and fed forward to downstream areas such as the SC. To our knowledge, there is no direct evidence for this assumption from the SEF (i.e. recording in a downstream structure during or after ICMSSEF), but many studies have reported greater oculomotor effects of stimulation to the SEF or the FEF when delivered at a presumed time of greater activity (Tehovnik et al., 1999; Gold \& Shadlen, 2000; Opris et al., 2001; Moore \& Armstrong, 2003; Chen \& Tehovnik, 2007); short-duration stimulation of the SC delivered later during a gap interval also evokes larger neck muscle responses, paralleling the level of endogenous SC activity at the time of stimulation (Corneil et al., 2007). While SC activity preceding ICMS-SEF is higher on pro- vs. anti-saccade trials (Everling et al., 1999), we suggest that the stimulation-evoked activity arising from ICMS-SEF drives the SC to a higher level of activity on anti-saccade trials. This would then feed down to the neck via a polysynaptic pathway, producing greater amounts of lateralized neck muscle recruitment on anti- vs. pro-saccade trials, despite the greater amount of baseline activity on pro-saccades. The lack of an accompanying evoked saccade would presumably be due to either the failure of stimulation-evoked activity within the SC to reach threshold or to not exceed threshold for a sufficient amount of time due to the very short duration of ICMS-SEF. Essentially, the evolution of a neck muscle response in the absence of a saccade arises from the selective inhibition of omni-pause neurons on saccadic, but not cephalomotor, elements (see above).

An alternative mechanism is required to explain the disruptive effects of ICMS-SEF on bilateral anti-saccade behavior. We surmise that such behavioral effects are manifest via a disruptive effect of ICMS-SEF on oculomotor activity that largely plays out after the cessation of stimulation. In Fig. 7, we illustrate this as a decrease in accumulating SEF and SC activity away from saccade threshold (as suggested by Kunimatsu \& Tanaka, 2012), with greater delays being present on anti- vs. pro-saccade trials given the larger role for the SEF in this behavior. In contrast to the feedforward and lateralized influence on neck muscle activity, we suggest that such disruption arises from feedback pathways, perhaps through the thalamus as noted above. Although data from the SEF is lacking, the FEF undergoes a large and prolonged period of hyperpolarization after electrical stimulation (Seidemann et al., 2002) that was suggested to involve the other, non-stimulated FEF. Whether this is also true of the SEF remains to be determined, but given the results of Seidemann and colleagues, a multiphasic response to ICMS within the SEF that consists of an initial excitation followed by a prolonged period of inhibitions seems plausible. One key prediction of our speculative mechanism is that such inhibition is itself state-dependent, being greater or perhaps more long-lasting on anti- vs. prosaccade trials. Disruption of the habitual evolution of SEF activity on anti-saccades would also increase the propensity of anti-saccade errors (not illustrated).

\section{Perspectives on state-dependency}

The diversity of effects evoked by ICMS-SEF provides a novel perspective on the effects of stimulation of a high-level area such as the SEF on behavior. ICMS-SEF can disrupt some aspects of oculomotor behavior while facilitating others, and future studies will need to determine whether the co-existence of disruptive and facilitative effects is unique to the SEF and to ICMS. In light of our results, functional interpretations based on state-dependent results should consider not only the direction of such influences (i.e. whether stimulation ostensibly disrupts or facilitates behavior), but also how such state-dependent results are assessed. To illustrate this, had we only looked at anti-saccade behavior, a plausible interpretation would be that ICMS selectively disrupts SEF processing for anti-saccades. Yet had we only looked at neck muscle recruitment, a plausible interpretation would have been that ICMS-SEF facilitates contralateral orienting for anti-saccades. Consideration of both sets of results reveals a more complete picture of a multiphasic response profile to ICMSSEF that plays out differently in different effectors. We surmise that the diversity of our results across multiple outputs reflects the richness of the feedforward and feedback connections of the SEF with other cortical and subcortical targets, emphasizing the highly complex and multiphasic influence of electrical microstimulation both within the SEF and throughout other interconnected networks.

\section{Acknowledgements}

This work was supported by operating grants from the Canadian Institutes of Health Research (MOP 93796, 120772) and a Discovery Grant from the Natural Sciences and Engineering Research Council of Canada (RGPIN 311680). B.B.C. was partially supported by an Ontario Graduate Scholarship. We thank Dr S. Cushing for the surgical implantation of the neck muscle electrodes and K. Green for expert technical and surgical assistance. The authors declare no competing financial interests.

\section{Abbreviations}

ACC, anterior cingulate cortex; DLPFC, dorso-lateral prefrontal cortex; EMG, electromyography; FEF, frontal eye field; FP, fixed point; ICMS-SEF, intracortical microstimulation to the supplementary eye field; OCI, obliquus capitis inferior; RCP maj, rectus capitis posterior major; RT, reaction time; SC, superior colliculus; SPL cap, splenius capitis.

\section{References}

Amador, N., Schlag-Rey, M. \& Schlag, J. (1998) Primate antisaccades. I. Behavioral characteristics. J. Neurophysiol., 80, 1775-1786.

Amador, N., Schlag-Rey, M. \& Schlag, J. (2004) Primate antisaccade. II. Supplementary eye field neuronal activity predicts correct performance. J. Neurophysiol., 91, 1672-1689.

Bell, A.H., Everling, S. \& Munoz, D.P. (2000) Influence of stimulus eccentricity and direction on characteristics of pro- and antisaccades in nonhuman primates. J. Neurophysiol., 84, 2595-2604.

Bernstein, N.A. (1967) The Coordination and Regulation of Movements. Pergamon Press, Oxford 
Chapman, B.B. \& Corneil, B.D. (2011) Neuromuscular recruitment related to stimulus presentation and task instruction during the anti-saccade task. Eur. J. Neurosci., 33, 349-360.

Chapman, B.B., Pace, M.A., Cushing, S.L. \& Corneil, B.D. (2012) Recruitment of a contralateral head turning synergy by stimulation of monkey supplementary eye fields. J. Neurophysiol., 107, 1694-1710.

Chen, L.L. \& Tehovnik, E.J. (2007) Cortical control of eye and head movements: integration of movements and percepts. Eur. J. Neurosci., 25, 1253-1264

Chen, L.L. \& Walton, M.M. (2005) Head movement evoked by electrical stimulation in the supplementary eye field of the rhesus monkey. J. Neurophysiol., 94, 4502-4519.

Cohen, M.R. \& Newsome, W.T. (2004) What electrical microstimulation has revealed about the neural basis of cognition. Curr. Opin. Neurobiol., 14, 169-177.

Corneil, B.D. (2011) Eye-head gaze shifts. In Liversedge, S.P., Gilchrist, I.D. \& Everling, S. (Eds), The Oxford Handbook of Eye Movements. Oxford University Press, Oxford, pp. 303-322

Corneil, B.D., Olivier, E., Richmond, F.J., Loeb, G.E. \& Munoz, D.P. (2001) Neck muscles in the rhesus monkey. II. Electromyographic patterns of activation underlying postures and movements. J. Neurophysiol., 86, 1729-1749.

Corneil, B.D., Olivier, E. \& Munoz, D.P. (2002) Neck muscle responses to stimulation of monkey superior colliculus. I. Topography and manipulation of stimulation parameters. J. Neurophysiol., 88, 1980-1999.

Corneil, B.D., Olivier, E. \& Munoz, D.P. (2004) Visual responses on neck muscles reveal selective gating that prevents express saccades. Neuron, 42, 831-841.

Corneil, B.D., Munoz, D.P. \& Olivier, E. (2007) Priming of head premotor circuits during oculomotor preparation. J. Neurophysiol., 97, 701-714.

Corneil, B.D., Munoz, D.P., Chapman, B.B., Admans, T. \& Cushing, S.L. (2008) Neuromuscular consequences of reflexive covert orienting. Nat. Neurosci., 11, 13-15.

Corneil, B.D., Elsley, J.K., Nagy, B. \& Cushing, S.L. (2010) Motor output evoked by subsaccadic stimulation of primate frontal eye fields. Proc. Natl. Acad. Sci. USA, 107, 6070-6075.

Elsley, J.K., Nagy, B., Cushing, S.L. \& Corneil, B.D. (2007) Widespread presaccadic recruitment of neck muscles by stimulation of the primate frontal eye fields. J. Neurophysiol., 98, 1333-1354.

Everling, S., Dorris, M.C., Klein, R.M. \& Munoz, D.P. (1999) Role of primate superior colliculus in preparation and execution of anti-saccades and pro-saccades. J. Neurosci., 19, 2740-2754.

Farshadmanesh, F., Chang, P., Wang, H., Yan, X., Corneil, B.D. \& Crawford, J.D. (2008) Neck muscle synergies during stimulation and inactivation of the interstitial nucleus of Cajal (INC). J. Neurophysiol., 100, 1677-1685.

Galiana, H.L. \& Guitton, D. (1992) Central organization and modeling of eye-head coordination during orienting gaze shifts. Ann. NY Acad. Sci., 656, 452-471.

Gandhi, N.J. \& Sparks, D.L. (2007) Dissociation of eye and head components of gaze shifts by stimulation of the omnipause neuron region. J. Neurophysiol., 98, 360-373.

Gerits, A., Ruff, C.C., Guipponi, O., Wenderoth, N., Driver, J. \& Vanduffel, W. (2011) Transcranial magnetic stimulation of macaque frontal eye fields decreases saccadic reaction time. Exp. Brain Res., 212, 143-152.

Gold, J.I. \& Shadlen, M.N. (2000) Representation of a perceptual decision in developing oculomotor commands. Nature, 404, 390-394.

Goonetilleke, S.C., Doherty, T.J. \& Corneil, B.D. (2010) A within-trial measure of the stop signal reaction time in a head-unrestrained oculomotor countermanding task. J. Neurophysiol., 104, 3677-3690.

Goonetilleke, S.C., Gribble, P.L., Mirsattari, S.M., Doherty, T.J. \& Corneil, B.D. (2011) Neck muscle responses evoked by transcranial magnetic stimulation of the human frontal eye fields. Eur. J. Neurosci., 33, 2155-2167.

Johnston, K. \& Everling, S. (2008) Neurophysiology and neuroanatomy of reflexive and voluntary saccades in non-human primates. Brain Cognition, 68, 271-283.

Johnston, K. \& Everling, S. (2011) Frontal cortex and flexible control of saccades. In Liversedge, S.P., Gilchrist, I.D. \& Everling, S. (Eds), The Oxford Handbook of Eye Movements. Oxford University Press, Oxford, UK, pp. 279-302.

Knight, T.A. (2012) Contribution of the frontal eye field to gaze shifts in the head-unrestrained rhesus monkey: neuronal activity. Neuroscience, 225, 213-236.

Kunimatsu, J. \& Tanaka, M. (2010) Roles of the primate motor thalamus in the generation of antisaccades. J. Neurosci., 30, 5108-5117.
Kunimatsu, J. \& Tanaka, M. (2012) Alteration of the timing of self-initiated but not reactive saccades by electrical stimulation in the supplementary eye field. Eur. J. Neurosci., 36, 3258-3268.

Martinez-Trujillo, J.C., Wang, H. \& Crawford, J.D. (2003) Electrical stimulation of the supplementary eye fields in the head-free macaque evokes kinematically normal gaze shifts. J. Neurophysiol., 89, 29612974.

Monteon, J.A., Avillac, M., Yan, X., Wang, H. \& Crawford, J.D. (2012) Neural mechanisms for predictive head movement strategies during sequential gaze shifts. J. Neurophysiol., 108, 2689-2707.

Moore, T. \& Armstrong, K.M. (2003) Selective gating of visual signals by microstimulation of frontal cortex. Nature, 421, 370-373.

Muri, R.M., Hess, C.W. \& Meienberg, O. (1991) Transcranial stimulation of the human frontal eye field by magnetic pulses. Exp. Brain Res., 86, 219223.

Nyffeler, T., Muri, R.M., Bucher-Ottiger, Y., Pierrot-Deseilligny, C., Gaymard, B. \& Rivaud-Pechoux, S. (2007) Inhibitory control of the human dorsolateral prefrontal cortex during the anti-saccade paradigm-a transcranial magnetic stimulation study. Eur. J. Neurosci., 26, 13811385.

Olk, B., Chang, E., Kingstone, A. \& Ro, T. (2006) Modulation of antisaccades by transcranial magnetic stimulation of the human frontal eye field. Cereb. Cortex, 16, 76-82.

Oommen, B.S., Smith, R.M. \& Stahl, J.S. (2004) The influence of future gaze orientation upon eye-head coupling during saccades. Exp. Brain Res., $155,9-18$.

Opris, I., Barborica, A. \& Ferrera, V.P. (2001) On the gap effect for saccades evoked by electrical microstimulation of frontal eye fields in monkeys. Exp. Brain Res., 138, 1-7.

Pascual-Leone, A., Walsh, V. \& Rothwell, J. (2000) Transcranial magnetic stimulation in cognitive neuroscience - virtual lesion, chronometry, and functional connectivity. Curr. Opin. Neurobiol., 10, 232-237.

Pélisson, D., Goffart, L., Guillaume, A., Catz, N. \& Raboyeau, G. (2001) Early head movements elicited by visual stimuli or collicular electrical stimulation in the cat. Vision Res., 41, 3283-3294.

Phillips, J.M., Johnston, K. \& Everling, S. (2011) Effects of anterior cingulate microstimulation on pro- and antisaccades in nonhuman primates. J. Cognitive Neurosci., 23, 481-490.

Rezvani, S. \& Corneil, B.D. (2008) Recruitment of a head-turning synergy by low-frequency activity in the primate superior colliculus. J. Neurophysiol., 100, 397-411.

Schlag, J. \& Schlag-Rey, M. (1987) Evidence for a supplementary eye field. J. Neurophysiol., 57, 179-200.

Schlag-Rey, M., Amador, N., Sanchez, H. \& Schlag, J. (1997) Antisaccade performance predicted by neuronal activity in the supplementary eye field. Nature, 390, 398-401.

Seidemann, E., Arieli, A., Grinvald, A. \& Slovin, H. (2002) Dynamics of depolarization and hyperpolarization in the frontal cortex and saccade goal Science, 295, 862-865.

Stuphorn, V. \& Schall, J.D. (2006) Executive control of countermanding saccades by the supplementary eye field. Nat. Neurosci., 9, 925-931.

Tehovnik, E.J., Slocum, W.M. \& Schiller, P.H. (1999) Behavioural conditions affecting saccadic eye movements elicited electrically from the frontal lobes of primates. Eur. J. Neurosci., 11, 2431-2443.

Terao, Y., Fukuda, H., Ugawa, Y., Hikosaka, O., Hanajima, R., Furubayashi, T., Sakai, K., Miyauchi, S., Sasaki, Y. \& Kanazawa, I. (1998) Visualization of the information flow through human oculomotor cortical regions by transcranial magnetic stimulation. J. Neurophysiol., 80, 936-946.

Valero-Cabre, A., Wattiez, N., Monfort, M., Francois, C., Rivaud-Pechoux, S., Gaymard, B. \& Pouget, P. (2012) Frontal non-invasive neurostimulation modulates antisaccade preparation in non-human primates. PLoS ONE, 7, e38674.

Watanabe, M. \& Munoz, D.P. (2010) Saccade suppression by electrical microstimulation in monkey caudate nucleus. J. Neurosci., 30, 2700-2709.

Watanabe, M. \& Munoz, D.P. (2011) Saccade reaction times are influenced by caudate microstimulation following and prior to visual stimulus appearance. J. Cognitive Neurosci., 23, 1794-1807.

Watanabe, M. \& Munoz, D.P. (2013) Effects of caudate microstimulation on spontaneous and purposive saccades. J. Neurophysiol., 110, 334-343.

Wegener, S.P., Johnston, K. \& Everling, S. (2008) Microstimulation of monkey dorsolateral prefrontal cortex impairs antisaccade performance. Exp. Brain Res., 190, 463-473.

Yang, S.N., Heinen, S.J. \& Missal, M. (2008) The effects of microstimulation of the dorsomedial frontal cortex on saccade latency. J. Neurophysiol., 99, $1857-1870$ 